\title{
Dietary Intake in Relation to the Risk of Reflux Disease: A Systematic Review
}

\author{
Neda Heidarzadeh-Esfahani ${ }^{1}$, Davood Soleimani ${ }^{2,3}$, Salimeh Hajiahmadi ${ }^{4}$, Shima Moradi ${ }^{5}$, \\ Nafiseh Heidarzadeh ${ }^{6}$, and Seyyed Mostafa Nachvak ${ }^{5}$ \\ ${ }^{1}$ Student Research Committee, School of Nutritional Sciences and Food Technology, ${ }^{2}$ Research Center of Oils and Fats, \\ ${ }^{3}$ Nutritional Sciences Department, School of Nutrition Sciences and Food Technology, and ${ }^{5}$ Department of Nutritional Sciences, \\ Research Center for Environmental Determinants of Health (RCEDH), Kermanshah University of Medical Sciences, \\ Kermanshah 6719851552, Iran \\ ${ }^{4}$ Department of Nutrition, School of Public Health, Shahid Sadoughi University of Medical Sciences, Yazd 8916188635, Iran \\ ${ }^{6}$ Depertment of Genetics, Faculty of Basic Sciences, Shahrekord University, Shahrekord 881863414, Iran
}

\begin{abstract}
Gastroesophageal reflux disease (GERD) is a chronic condition which has a high global prevalence. Dietary intake is considered to be a contributing factor for GERD. However, scientific evidence about the effect of diet on the risk of GERD is controversial. This systematic review was conducted to address this issue. A comprehensive structured search was performed using the MEDLINE, Scopus, and Web of Science databases up to August 2020, in accordance with the PRISMA statement. No restrictions were set in terms of language, time of publication, or study location. Study selection and data abstraction was conducted independently by two authors, and risk of bias was assessed using a modified Quality in Prognosis Studies Tool. Eligible studies evaluating the impact of food and dietary pattern on GERD were included in qualitative data synthesis. After excluding duplicate, irrelevant, and low quality studies, 25 studies were identified for inclusion: 5 case-control studies, 14 cross-sectional studies, and 6 prospective studies. This review indicates that high-fat diets, carbonated beverages, citrus products, and spicy, salty, and fried foods are associated with risk of GERD.
\end{abstract}

Keywords: diet, food, gastroesophageal reflux, systematic review

\section{INTRODUCTION}

Gastroesophageal reflux disease (GERD) is a common disorder that affects quality of life. GERD develops when reflux of stomach contents causes troublesome symptoms and long-term complications (Rajaie et al., 2020). The major symptoms of GERD include heartburn and regurgitation (Kahrilas, 2003), however, GERD can also manifest with atypical symptoms including epigastric pain, dyspepsia, nausea, bloating, and belching (Badillo and Francis, 2014). GERD pathogenesis involves esophagitis, hemorrhage, stricture, Barrett's esophagus, and adenocarcinoma (Rajaie et al., 2020). Moreover, GERD is independently associated with increased risk of cardiovascular diseases, including acute myocardial infarction (Lei et al., 2017). It is a global disease, with an estimated highest incidence in North America (18.1\% 27.8\%), followed by the Middle-East America (8.7\% 33.1\%),
Europe $(8.8 \% \sim 25.9 \%)$, and East Asia $(2.5 \% \sim 7.8 \%)$ (Seremet et al., 2015).

GERD is a multifactorial disease influenced by both genetic predisposition and environmental factors. Diet (an environmental factor) has important roles in gastrointestinal and cardio metabolic disorders (Argyrou et al., 2018; Heshmati et al., 2019; Surdea-Blaga et al., 2019; Heshmati et al., 2020), and modifiable risk factors included long meal-to-sleep intervals, speed of eating, and scale and temperature of foods (Esmaillzadeh et al., 2013; Yuan et al., 2017). Intake of alcohol, chocolate, and highfat meals reduces esophageal sphincter pressure and increases esophageal exposure to gastric juices (Kaltenbach et al., 2006). Some studies have reported that both the quality and quantity of carbohydrates in diet may be associated with GERD (Keshteli et al., 2017; Wu et al., 2018). However, current data are contradictory (Kim et al., 2014). 
Emerging data indicates that appropriate eating behaviors, i.e., healthy diets involving high intakes of fruits and whole grains (Wu et al., 2013), such as the Mediterranean diet (Mone et al., 2016), improves GERD symptoms. Therefore, improving diets can decrease the occurrence of GERD and should be considered a cost-effective strategy instead of pharmacotherapy.

Review studies have investigated predictors of GERD risk in terms of food related factors such as probiotics (Cheng and Ouwehand, 2020) and food components (Surdea-Blaga et al., 2019). However, to our knowledge, no systematic reviews have been conducted to assess the impact of diet on risk of reflux disease. We conducted a systematical review of articles investigating the association between food and dietary patterns with GERD.

\section{MATERIALS AND METHODS}

\section{Search strategy}

The literature search was conducted by two independent researchers using electronic databases, including the Web of Sciences, PubMed/MEDLINE, and Scopus, to identify relevant publications up to August 2020. This study was given ethical approval by the Ethics Committee of Research Council of Kermanshah University of Medical Sciences (Ethics Code: IR.KUMS.REC.1399.941).

We performed the systematic search using Medical Sub- ject Headings (MeSH) along with non-MeSH keywords in the title and abstract as follows: "Diet" OR "Food" OR "Dietary Pattern" OR "Food Pattern" AND "Gastroesophageal Reflux" OR "Gastric Acid Reflux" OR "Gastroesophageal Reflux Disease" OR "GERD” OR "Esophageal Reflux" OR "Pyrosis" OR "Pyroses" OR "Heartburn" OR "Barrett's Esophagus". We did not consider any restrictions in terms of language, time of publication, and study location.

\section{Inclusion and exclusion criteria}

Eligible studies were performed on adults and evaluated all components of the dietary patterns and risk of reflux disease. In addition, we considered all observational studies, including cross-sectional, case-control, prospective, and retrospective studies. Interventional studies were not included in this study since the duration of exposure was short. Overall, 991 articles were identified during the initial search and duplicate studies $(n=26)$ were removed. The remaining studies were screened based on topic and 833 irrelevant studies were excluded. Thereafter, 119 studies were reviewed in more detail, and 24 classed as irrelevant (including 17 that did not assess dietary pattern) and 52 that did not evaluate our outcome ("reflux diseases") were excluded. In addition, the qualification of 26 articles were evaluated and one study was excluded because of low quality. In total, 25 articles were eligible for inclusion in this review study (Fig. 1).

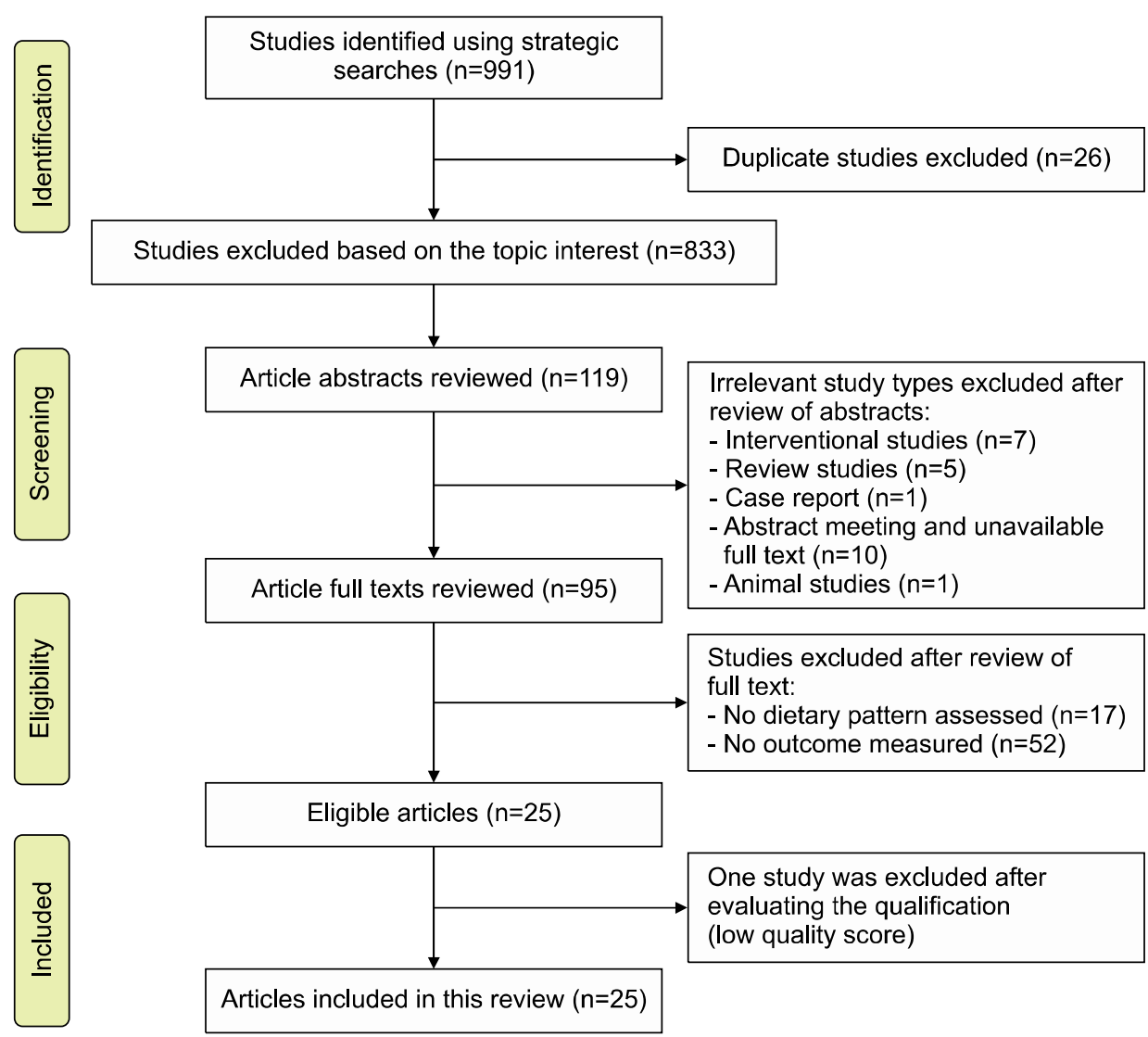

Fig. 1. Flow chart of the search and publication selection. 


\section{Quality assessment}

Using all the data extracted, we scored the risk of bias of the selected studies on a six-point scale using a modified version of the Quality in Prognosis Studies (Hayden et al., 2006). Using this system, we assessed the quality of individual studies using the following criteria (one point per criterion): (I) study participation (the study sample represents the key characteristics of the population of interest sufficiently well to limit potential bias to the results); (II) study attrition (loss to follow-up is not associated with key characteristics); (III) prognostic factor measurement (prognostic factors of interest are measured in study participants in such a way that potential bias is limited); (IV) confounding measurement and account (outcomes of interest are measured in study participants in such a way that potential bias is limited); (V) outcome measurement (important potential confounders are appropriately accounted for, limiting potential bias with respect to the prognostic factor of interest); and (VI) analysis (the statistical analysis is appropriate for the design of the study, and limits the potential for invalid results). Studies with a score between 0 and 3 points were considered to be of low quality, while studies with a score $>3$ to 6 were considered to be of high quality.

\section{Data extraction}

Data extraction was performed independently by two researchers using a data collection checklist. Any disagreement has been discussed and resolved accordingly. For each article, the first author's name, publication year, study design (trial/prospective/cross-sectional/case control), sample size, study population demographics [age, sex, body mass index (BMI), and country], dietary assessment tools, dietary components, and outcomes (all reported data on association between GERD and dietary components) were extracted. All data are presented in the Results.

\section{RESULTS}

\section{Study characteristics}

In this literature review, we identified 25 eligible studies with quality scores from 3.5 to 6.0. As shown in Table 1, these studies included 5 case-control studies (Nandurkar et al., 2004; Murphy et al., 2010; Wu et al., 2013; Asl et al., 2015; Ebrahimi-Mameghani et al., 2017), 14 crosssectional studies (El-Serag et al., 2005a; El-Serag et al., 2005b; Shapiro et al., 2007; Friedenberg et al., 2010; Kubo et al., 2014; Khodarahmi et al., 2016; Mone et al., 2016; Alkhathami et al., 2017; Eslami et al., 2017; Keshteli et al., 2017; Atta et al., 2019; Kim et al., 2019; Kariri et al., 2020; Rajaie et al., 2020), and 6 prospective studies (Ruhl and Everhart, 1999; Gutschow et al., 2005;
Austin et al., 2006; Bhatia et al., 2011; López-Colombo et al., 2017; Wu et al., 2018). There were a total of 8 to 12,349 subjects per study. Studies were conducted in America (Ruhl and Everhart, 1999; Nandurkar et al., 2004; El-Serag et al., 2005a; El-Serag et al., 2005b; Austin et al., 2006; Shapiro et al., 2007; Friedenberg et al., 2010; Kubo et al., 2014; López-Colombo et al., 2017), Asia (Bhatia et al., 2011; Wu et al., 2013; Asl et al., 2015; Khodarahmi et al., 2016; Alkhathami et al., 2017; Ebrahimi-Mameghani et al., 2017; Eslami et al., 2017; Keshteli et al., 2017; Wu et al., 2018; Atta et al., 2019; Kim et al., 2019; Kariri et al., 2020; Rajaie et al., 2020), and Europe (Gutschow et al., 2005; Murphy et al., 2010; Mone et al., 2016). All studies included participates aged $\geq 18$ years of age, and most assessed dietary intake using the food frequency questionnaire.

\section{Study outcomes}

A total of nine studies examined the negative effects of reflux-triggering foods, including high-fat, spicy, fried, and citrus foods, carbonated beverages, and tea (Shapiro et al., 2007; Kubo et al., 2014; Asl et al., 2015; Alkhathami et al., 2017; Eslami et al., 2017; López-Colombo et al., 2017; Atta et al., 2019; Kim et al., 2019; Kariri et al., 2020). Other food components, such as saturated fatty acids (SFA), monounsaturated fatty acids, polyunsaturated fatty acids, cholesterol, smoky foods, salty foods, coffee, alcohol, chocolate, and dairies, did not contribute to risk of GERD (Nandurkar et al., 2004; El-Serag et al., 2005a; El-Serag et al., 2005b; Shapiro et al., 2007; Friedenberg et al., 2010; Murphy et al., 2010; Bhatia et al., 2011; Wu et al., 2013; Kubo et al., 2014; Asl et al., 2015; Alkhathami et al., 2017; Eslami et al., 2017; López-Colombo et al., 2017; Atta et al., 2019). The results of studies examining the role of dietary components in reflux disease are shown in Table 2.

Shapiro et al. (2007), Kubo et al. (2014), Asl et al. (2015), and Kim et al. (2019) observed that high-fat diets contributed to the risk of reflux disease. However, Ruhl and Everhart (1999) and Wu et al. (2013) did not observe any association between high-fat diets and reflux disease. On the other hand, Ruhl and Everhart (1999) reported that cholesterol may increase the risk of reflux disease, and El-Serag et al. (2005a; 2005b) found the relationship between dietary fat and reflux disease was nonsignificant after adjusting for BMI, energy intake, and demographic characteristics. Moreover, daily intakes of total fat, SFA, cholesterol, energy from dietary fat, and fat were significantly higher in subjects without GERD symptoms than those with GERD symptoms.

Fast food is a possible causal risk factor for reflux disease (Alkhathami et al., 2017; Kariri et al., 2020). Indeed, Kubo et al. (2014) and Atta et al. (2019) found a significant relationship between fried foods and symptoms of 


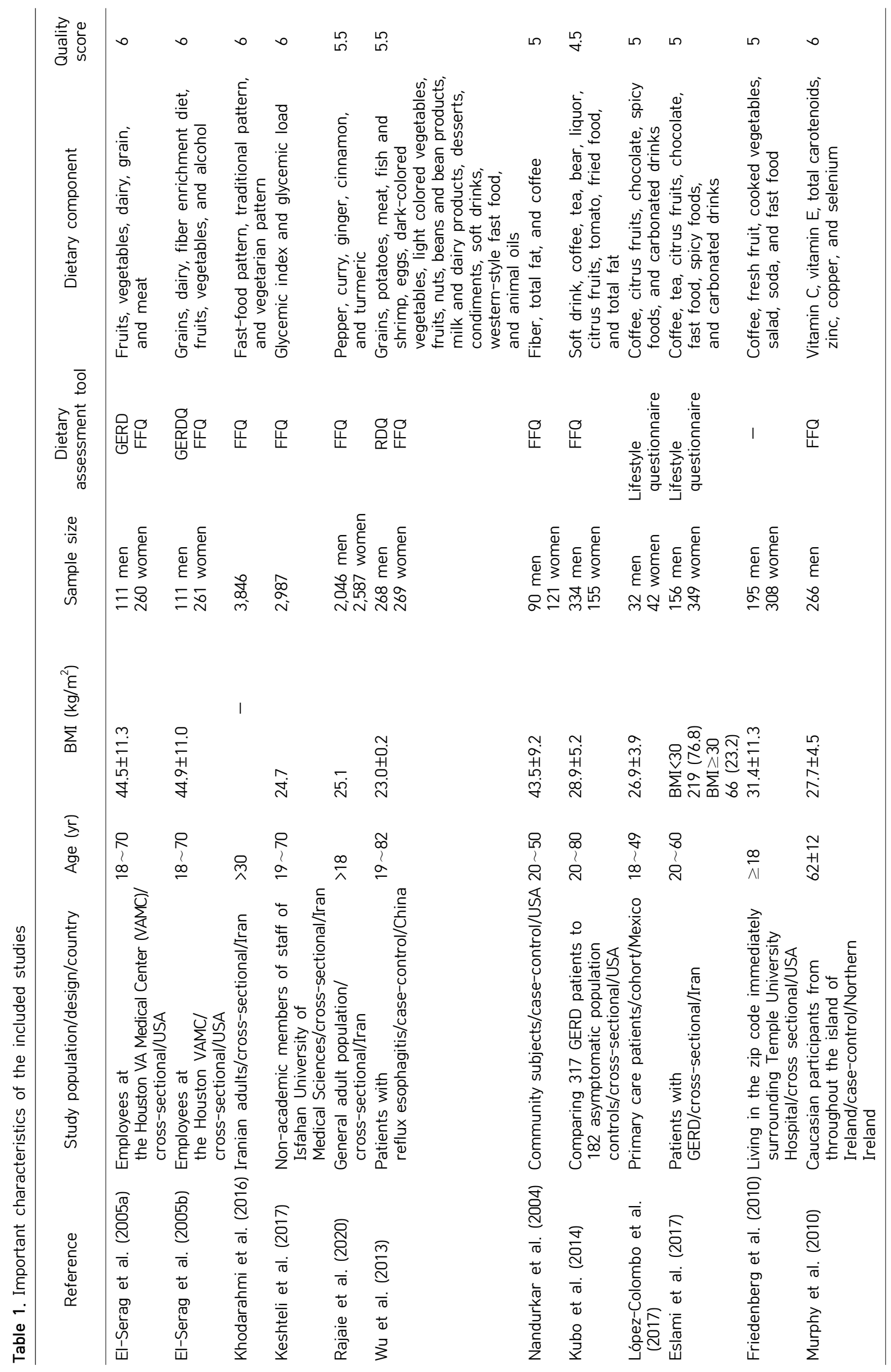




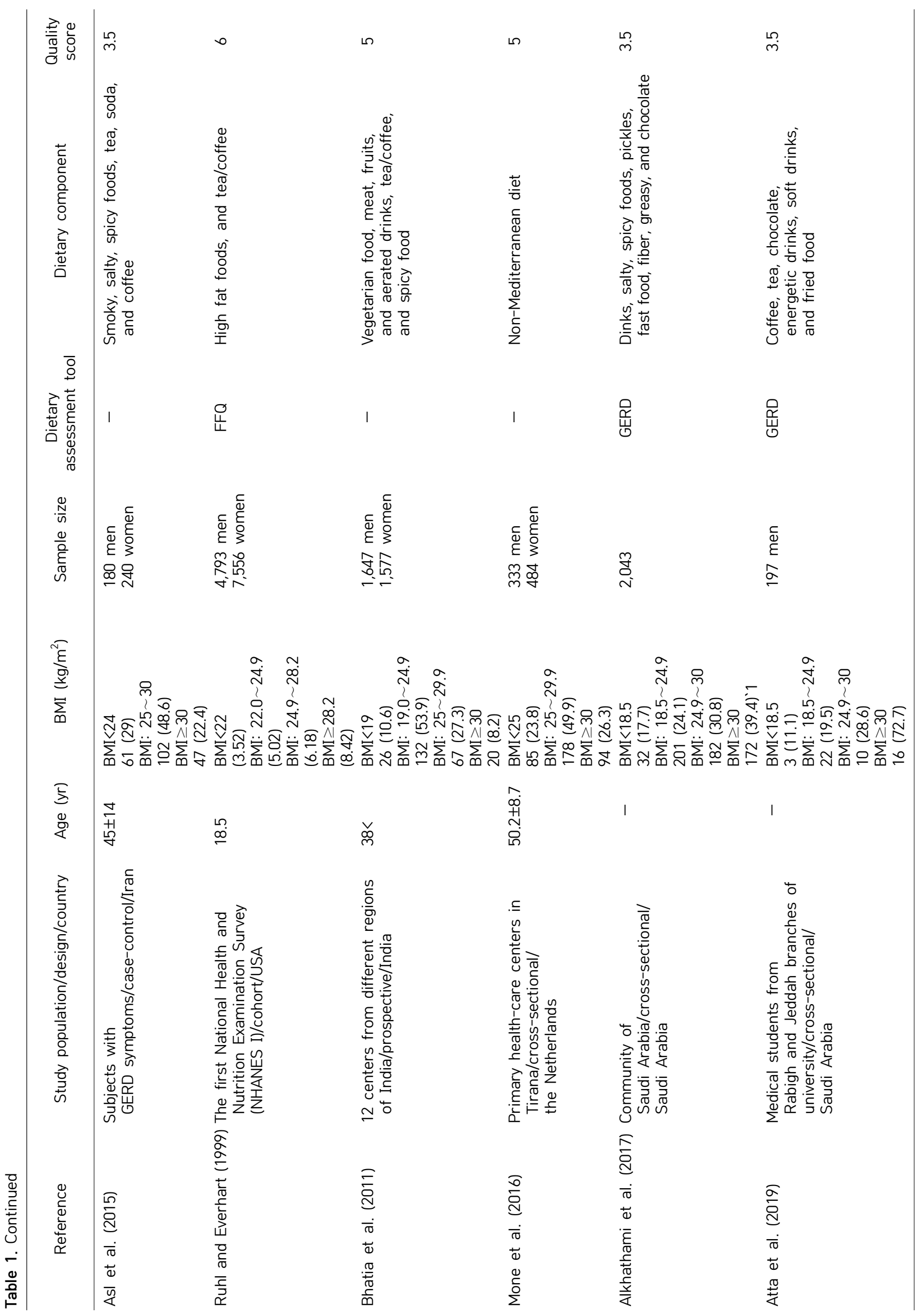




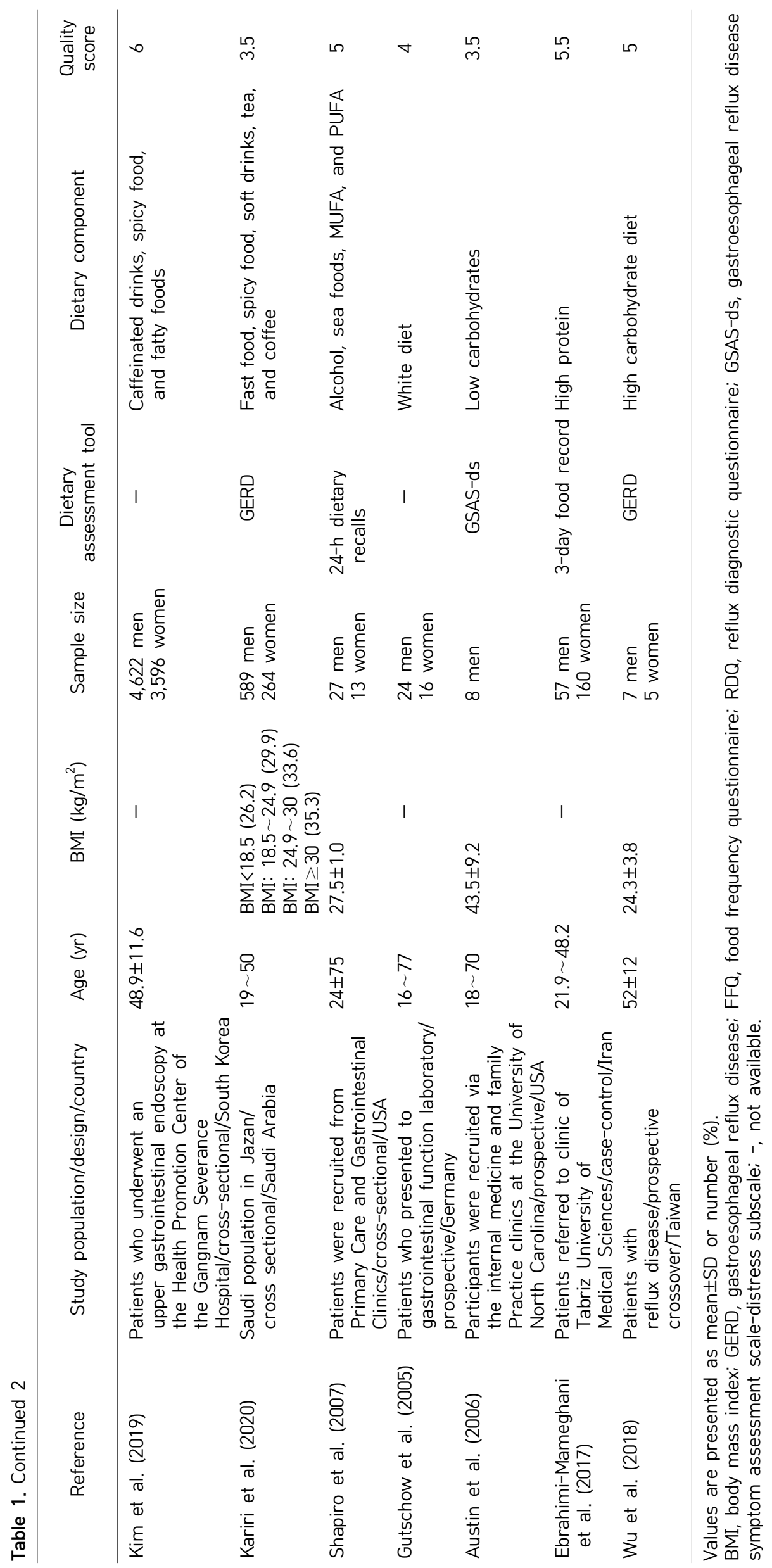




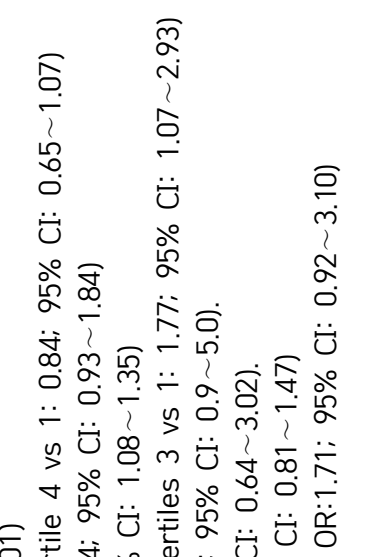

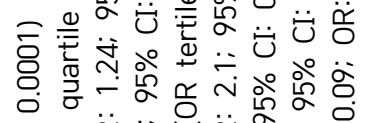

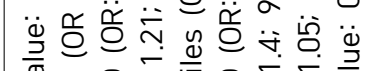

은

음

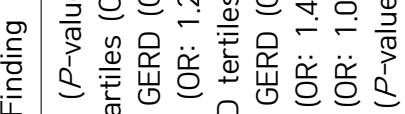

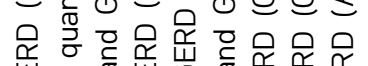

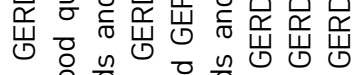

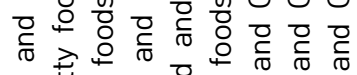

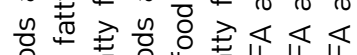

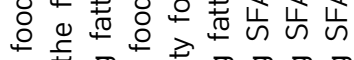

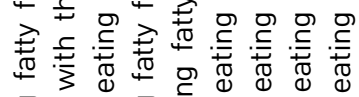

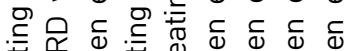

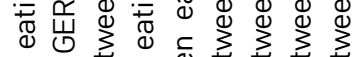
ᄃ

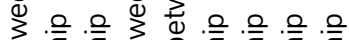

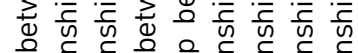

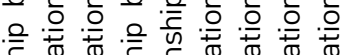

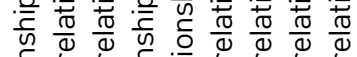

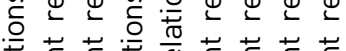

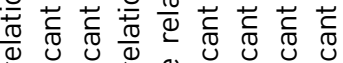

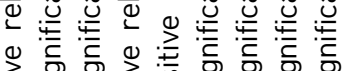

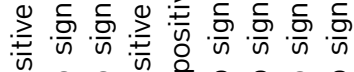

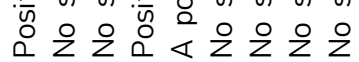

ลำ

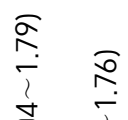

$\therefore$

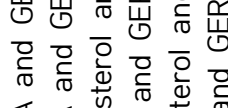

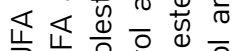

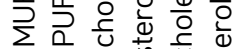

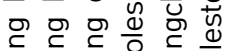

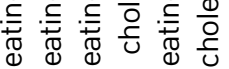

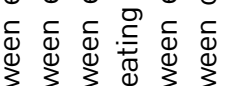

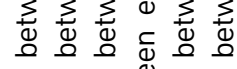
을 을 을 कि

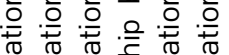

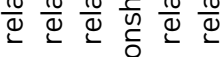

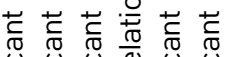

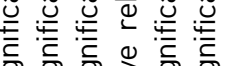

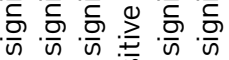

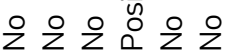

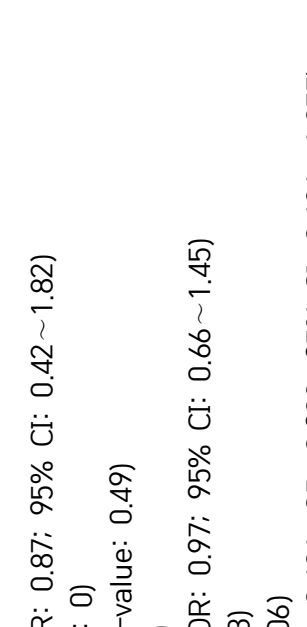

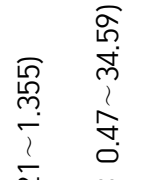

ब。

$\underset{\substack{\sigma \\ \infty}}{\infty}$.

ষ্ণ

is

लु.

$\ddot{U} \ddot{-}$

ㅇํㅇㅇำ

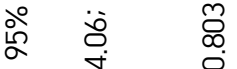

ذั

宛

a مूं

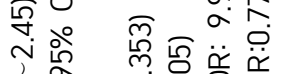

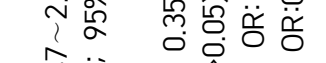

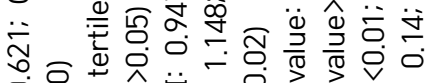
它

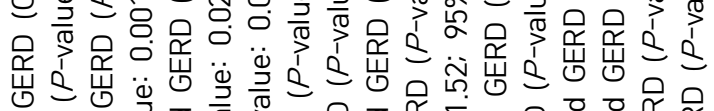

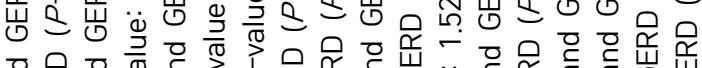

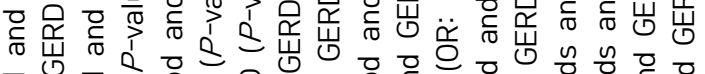
等

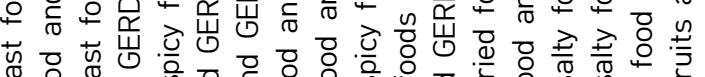

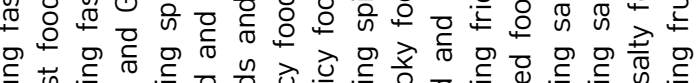

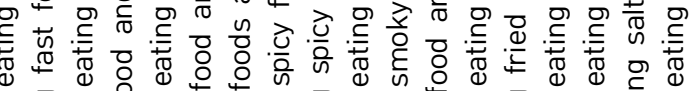

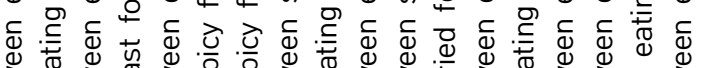

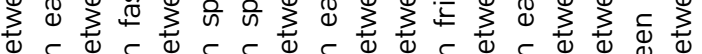
ه

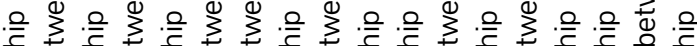

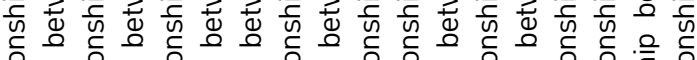

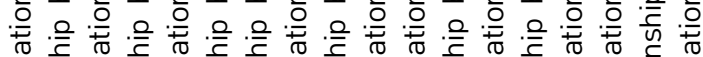
Q

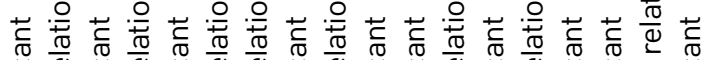

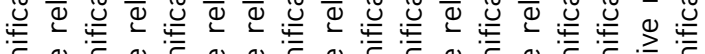

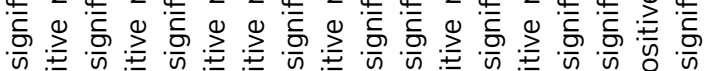

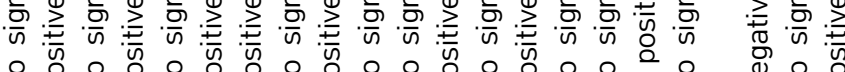

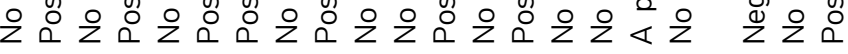

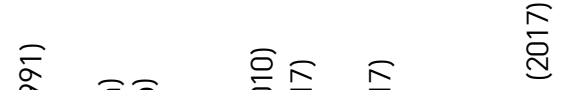

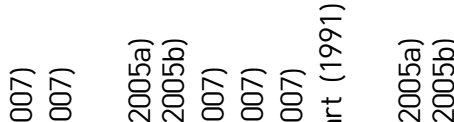

을

总 ஜิ̊ 宅乎

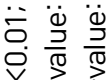

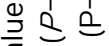

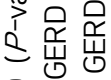
足点 을 의 要 要

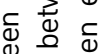

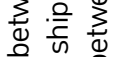
을 을 을

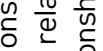
范范竞 Q 要 ज :

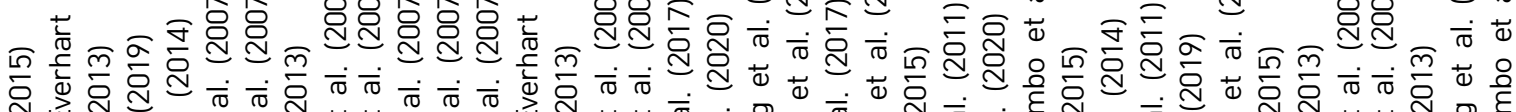

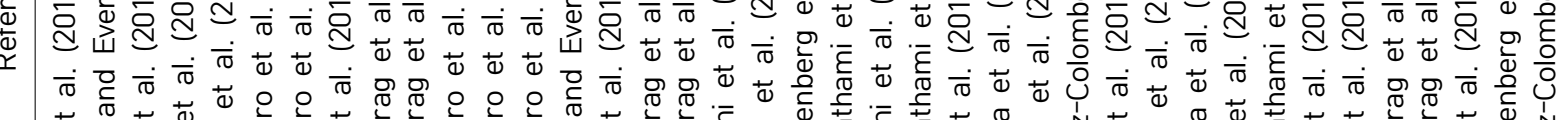
屯

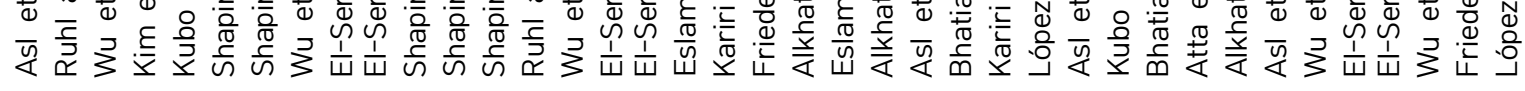




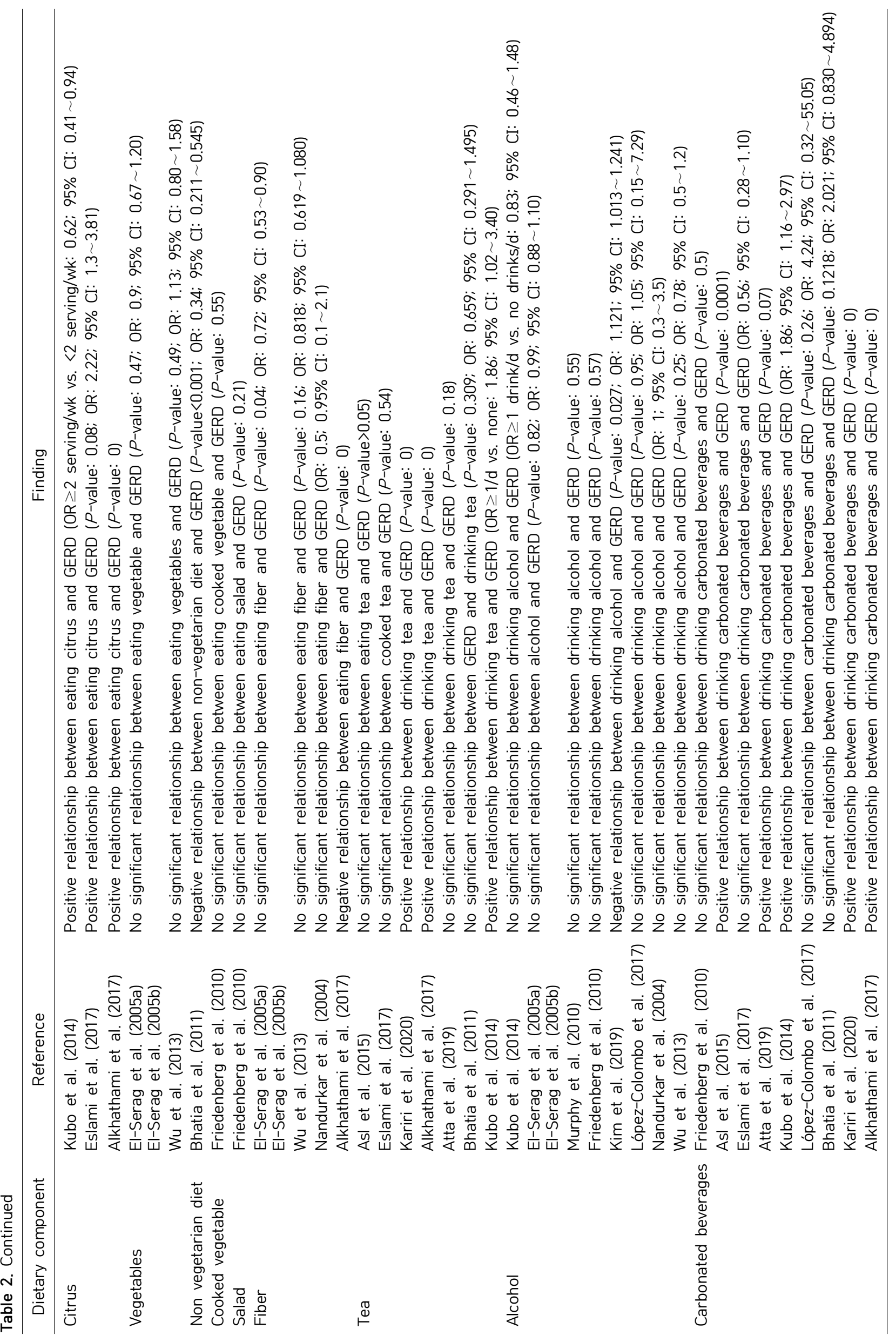




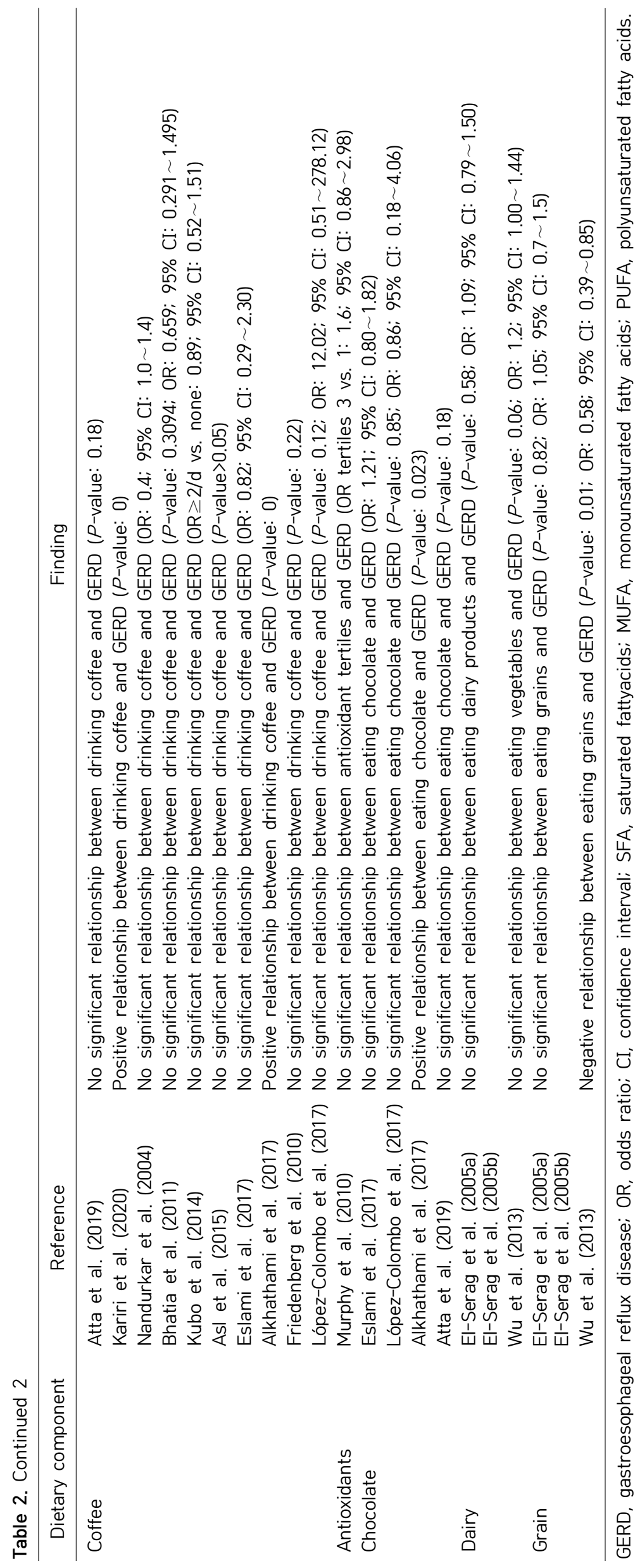




\section{GERD.}

Keshteli et al. (2017) showed that foods with high-glycemic indexes increase the risk of uninvestigated heartburn [odd ratio $(\mathrm{OR}=1.75 ; 95 \%$ confidence interval $(\mathrm{CI})$ : 1.03, 2.97; $P$-value $=0.04)]$ and uninvestigated chronic dyspepsia $(\mathrm{OR}=2.14 ; 95 \% \mathrm{CI}$ : $1.04,4.37$; $P$-value $=0.04)$ in men but not in women, even after adjusting for potential confounders, such as age, marital status, medications, education, sleeping, eating rate, and intake of spicy foods, cocoa, sugar-sweetened beverages, tea, coffee, energy, fat, fructose, and fiber. However, Kubo et al. (2014), Asl et al. (2015), Alkhathami et al. (2017), Atta et al. (2019), and Kariri et al. (2020) found a significant relationship between reflux disease and carbonated beverages, including soda, aerated, and soft drinks.

$\mathrm{Wu}$ et al. (2013) observed a negative relationship between intake of fruits and reflux disease, whereas other studies did not observe any significant association (Friedenberg et al., 2010; El-Serag et al., 2005a; El-Serag et al., 2005b). Kubo et al. (2014), Alkhathami et al. (2017), Eslami et al. (2017), and López-Colombo et al. (2017) found that citrus was associated with risk of GERD. Moreover, consumption of non-vegetarian foods was an independent predictor of GERD (Bhatia et al., 2011). However, El-Serag et al. (2005a; 2005b) and Wu et al. (2013) did not observe any association between vegetables and GERD.

Two studies reported that fast foods, including sausage, fried chicken, pizza, hamburgers, french fries, and doughnuts, were not significantly associated with reflux disease (Friedenberg et al., 2010; Eslami et al., 2017). Alkhathami et al. (2017) reported that the prevalence of GERD was higher in those who did not consume dietary fibers regularly.

\section{DISCUSSION}

To the best of our knowledge, this is the first systematic review to investigate the relationship between different foods and dietary patterns with the occurrence of GERD. Unlike previous review studies, the present study reviewed all previous observational studies. The results of this study showed a significant association between adherences to high-fat diets and increased the risk of GERD.

Consumption of large high-fat meals appears to accelerate development of GERD (Surdea-Blaga et al., 2019) by reducing lower esophageal sphincter (LES) pressure (Kumar and Katz, 2013; Kubo et al., 2014; Asl et al., 2015). Furthermore, large high-fat meals are correlated with increased acid exposure time in patients compared with low fat meals (Kahrilas et al., 2008; Kubo et al., 2010; Castillo et al., 2015; Ireland et al., 2016; Sethi and Richter, 2017). However, previous review studies in 2000 and 2009 investigating the pathogenic relationship between eating habits and occurrence of GERD were unable support the effect of dietary fat on incidence of GERD (Meining and Classen, 2000; Festi et al., 2009). In contrast to the results of the present study, Eslick and Talley (2009) emphasized the relationship between high cholesterol consumption and increased risk of GERD. The authors concluded that confounding factors such as BMI, energy and demographic variables were responsible for this result.

Decreased LES pressure is involved in progression of GERD in overweight individuals consuming a high-cholesterol diet. However, further studies are needed to investigate the independent association of dietary fat as a risk factor for GERD. Several studies reported that consuming a high-fat diet and food rich in cholesterol and SFA (El-Serag et al., 2005a; El-Serag et al., 2005b; Shapiro et al., 2007; Wu et al., 2013), and unsaturated fatty acids (Shapiro et al., 2007) was not associated with increased risk of GERD. Similarly, review studies investigating the pathogenic relationship between eating habits and occurrence of GERD in 2000 (Meining and Classen, 2000) and 2009 (Festi et al., 2009) did not support an effect of dietary fat on the incidence of GERD.

Consuming high-salt foods (Asl et al., 2015; Alkhathami et al., 2017), spicy foods (Bhatia et al., 2011; Eslami et al., 2017; López-Colombo et al., 2017), smoky foods (Asl et al., 2015), and fast foods (Friedenberg et al., 2010; Eslami et al., 2017) does not significantly increase the risk of GERD. However, several studies have emphasized the relationship between consumption of high-salt foods (Wu et al., 2013) and high-spice foods (Asl et al., 2015; Alkhathami et al., 2017; Kariri et al., 2020) in accelerating development of GERD. Furthermore, Wu et al. (2013) showed that increasing salt intake is only effective in reducing LES pressure, and alone cannot increase the risk of GERD. In addition, in Asian populations, consuming high-spice foods followed by the habit of lying down after eating increases the risk of GERD. Lying down after eating may be a major cause of this disorder since it reduces LES pressure and affects the reflux of gastric contents (Asl et al., 2015).

Vegetables (cooked vegetables and salad) (El-Serag et al., 2005a; El-Serag et al., 2005b; Friedenberg et al., 2010; Wu et al., 2013), dietary fiber (Nandurkar et al., 2004; El-Serag et al., 2005a; El-Serag et al., 2005b; Wu et al., 2013), dairy products (El-Serag et al., 2005a; El-Serag et al., 2005b; Wu et al., 2013), and antioxidants (Murphy et al., 2010) were also not significantly associated with increased incidence of GERD. A previous review study investigating the relationship between diet and GERD showed that adherence to the Mediterranean diet (rich in vegetables, fiber, and antioxidants) (Winberg et al., 2012), could play a preventive role in GERD, especially 
among patients with underlying diseases such as diabetes, heart disease, and cancer (Badillo and Francis, 2014; Newberry and Lynch, 2017). However, these results are inconsistent with those of the present study. Further studies are needed to assess the effectiveness of these food groups in preventing GERD in all individuals.

Consumption of high-caffeine products such as tea (Asl et al., 2015; Eslami et al., 2017), coffee (Nandurkar et al., 2004; Friedenberg et al., 2010; Kubo et al., 2014; Asl et al., 2015; Eslami et al., 2017; López-Colombo et al., 2017), and chocolate (Eslami et al., 2017; López-Colombo et al., 2017) are also not significantly associated with risk of developing GERD. Review studies investigating the relationship between lifestyle and GERD also did not support a role of high-caffeine sources in increasing the incidence of GERD (Meining and Classen, 2000; Kaltenbach et al., 2006; Vemulapalli, 2008). However, in 2014, a study suggested that tea consumption was associated with increased risk of GERD. These contradictory results may be due to differences between the types of tea consumed by the subjects in the study (Kubo et al., 2014). In addition, some studies have shown that coffee relaxes the LES, and increases percentage reflux time in the fasting state (Akbar and Howden, 2016).

Similarly to our results, other studies have not shown a significant relationship between alcohol consumption and increased risk of GERD (Festi et al., 2009; Kubo et al., 2010; Esmaillzadeh et al., 2013; Ireland et al., 2016). A further review study was conducted in parallel with our own, which showed that consumption of carbonated beverages increases the risk of GERD (Newberry and Lynch, 2017). Carbonated beverages may increase the likelihood of dysphagia reflexes by altering the acidity of the gastrointestinal tract, especially the stomach, and affecting digestion (intragastric residence time and inducing poor digestion) (Asl et al., 2015). Furthermore, these beverages contain high levels of acidity, added sugars and artificial sweeteners, and caffeine, which alter LES pressures and intraesophageal $\mathrm{pH}$ (Newberry and Lynch, 2017).

The results of the present study show that daily consumption of citrus increases risk of GERD (López-Colombo et al., 2017), which is consistent with the results of prior review studies (Meining and Classen, 2000; Kaltenbach et al., 2006; Sethi and Richter, 2017). These fruits increase the risk of GERD by reducing LES pressure or delayed gastric emptying (Eslami et al., 2017). A previous study conducted on adherence to dietary recommendations in GERD (Kubo et al., 2014) showed that frequent consumption of citrus fruits and juices of adjusted $\mathrm{pH}$ plays a preventive role in acid-sensitive individuals. Other compounds in citrus fruits in addition to acidity are also likely to play a preventive role. However, this study attributed infrequent citrus fruit intake to the strong recommendations on limiting these fruits in people predisposed to dysphagia, and did not provide any documented data linking this group of fruits to GERD (Kubo et al., 2014).

In the present study, a diet rich in fruits did not significantly effect on impact the risk of developing GERD. Nevertheless, results from previous review studies support the protective effect of fruits in reducing the incidence of reflux disorders (Wu et al., 2013; Badillo and Francis, 2014). Indeed, these studies attribute this positive effect to the high-fiber content in fruits (Badillo and Francis, 2014). Moreover, consumption of fruit juices increases pressure the gradient from the abdomen to the chest, resulting in GERD symptoms by increasing abdominal pressure or decreasing LES pressure (Fallah et al., 2020). Further studies are needed to investigate the relationship between different compounds in fruits and GERD.

In parallel with the current study, a review study was conducted in 2017 that showed whole grain consumption is significantly correlated with reduced incidence of GERD in people with underlying diseases such as diabetes, heart disease, and cancer (Badillo and Francis, 2014). Further studies are needed to evaluate the effectiveness of grain consumption in reducing the incidence of this disorder in all individuals. The inconsistencies between studies may arise from differences in the socio-demographic statuses of the study populations, sample sizes, and criteria used to diagnose reflux disease.

Review studies have several inherent limitations that should be considered. First, although the present study investigated the relationship between different food groups and GERD in adults of different races and in different geographical conditions by extracting data from observational studies, it was not possible to investigate different age groups due to data limitations. Hence, we recommend other researchers conduct systematic review of observational and clinical trial studies investigating the effect of diet on GERD in children. Second, there were significant heterogeneity between studies due to varying regimens, doses, duration, center settings, and populations. Hence, we could not determine the fundamental factors to explain the observed heterogeneity due to the limited number of studies and lack of information for pooling. Third, it is crucial to investigate grey literature as an important resource in systematic review in order to reduce publication bias, which was unfortunately neglected from the current study.

In this study, we concluded that diets rich in vegetables, fiber, antioxidants, and caffeine were not significantly associated with increased risk of dysphagia. However, consumption of citrus fruits, carbonate beverages, spicy, and fried food increases the risk of developing this disorder. Furthermore, we did not identify a specific diet that plays 
an effective role in GERD. Other large-scale studies with robust study designs are needed to investigate the effect of different diets associated with this disorder in all age groups.

\section{AVAILABILITY OF DATA AND MATERIALS}

The datasets used and analyzed during the present study are available from the corresponding author on reasonable request.

\section{ACKNOWLEDGEMENTS}

We thank gratefully acknowledge the Research Council of Kermanshah University of Medical Sciences.

\section{FUNDING}

This research was funded by Research Council of Kermanshah University of Medical Sciences, Kermanshah, Iran (Grant No: 3010879).

\section{AUTHOR DISCLOSURE STATEMENT}

The authors declare no conflict of interest.

\section{REFERENCES}

Akbar A, Howden CW. Lifestyle modifications in GERD. In: Vaezi MF, editor. Diagnosis and Treatment of Gastroesophageal Reflux Disease. Springer, Cham, Switzerland. 2016. p 59-70.

Alkhathami AM, Alzahrani AA, Alzhrani MA, Alsuwat OB, Mahfouz MEM. Risk factors for gastroesophageal reflux disease in Saudi Arabia. Gastroenterology Res. 2017. 10:294-300.

Argyrou A, Legaki E, Koutserimpas C, Gazouli M, Papaconstantinou I, Gkiokas G, et al. Risk factors for gastroesophageal reflux disease and analysis of genetic contributors. World J Clin Cases. 2018. 6:176-182.

Asl SF, Mansour-Ghanaei F, Samadi H, Joukar F. Evaluations of life style factors and the severity of gastroesophageal reflux disease; a case-control study. Int J Mol Epidemiol Genet. 2015. 6:27-32.

Atta MM, Sayed MH, Zayed MA, Alsulami SA, Al-Maghrabi AT, Kelantan AY. Gastro-oesophageal reflux disease symptoms and associated risk factors among medical students, Saudi Arabia. Int J Gen Med. 2019. 12:293-298.

Austin GL, Thiny MT, Westman EC, Yancy WS Jr, Shaheen NJ. A very low-carbohydrate diet improves gastroesophageal reflux and its symptoms. Dig Dis Sci. 2006. 51:1307-1312.

Badillo R, Francis D. Diagnosis and treatment of gastroesophageal reflux disease. World J Gastrointest Pharmacol Ther. 2014. 5: 105-112.

Bhatia SJ, Reddy DN, Ghoshal UC, Jayanthi V, Abraham P, Choudhuri G, et al. Epidemiology and symptom profile of gas- troesophageal reflux in the Indian population: report of the Indian Society of Gastroenterology Task Force. Indian J Gastroenterol. 2011. 30:118-127.

Castillo R, Otero W, Trespalacios A. Evidence based review of the impact of treatments of gastroesophageal reflux disease. Rev Col Gastroenterol. 2015. 30:427-442.

Cheng J, Ouwehand AC. Gastroesophageal reflux disease and probiotics: a systematic review. Nutrients. 2020. 12:132.

Ebrahimi-Mameghani M, Sabour S, Khoshbaten M, Arefhosseini SR, Saghafi-Asl M. Total diet, individual meals, and their association with gastroesophageal reflux disease. Health Promot Perspect. 2017. 7:155-162.

El-Serag HB, Graham DY, Satia JA, Rabeneck L. Obesity is an independent risk factor for GERD symptoms and erosive esophagitis. Am J Gastroenterol. 2005a. 100:1243-1250.

El-Serag HB, Satia JA, Rabeneck L. Dietary intake and the risk of gastro-oesophageal reflux disease: a cross sectional study in volunteers. Gut. 2005b. 54:11-17.

Eslami O, Shahraki M, Bahari A, Shahraki T. Dietary habits and obesity indices in patients with gastro-esophageal reflux disease: a comparative cross-sectional study. BMC Gastroenterol. 2017. 17:132. https://doi.org/10.1186/s12876-017-0699-1

Eslick GD, Talley NJ. Gastroesophageal reflux disease (GERD): risk factors, and impact on quality of life-a population-based study. J Clin Gastroenterol. 2009. 43:111-117.

Esmaillzadeh A, Keshteli AH, Feizi A, Zaribaf F, Feinle-Bisset C, Adibi P. Patterns of diet-related practices and prevalence of gastro-esophageal reflux disease. Neurogastroenterol Motil. 2013. 25:831-e638.

Fallah Z, Ferns GA, Ghayour-Mobarhan M. Fluid intake and functional gastrointestinal disease: a narrative review. Crit Comments Biomed. 2020. 1:e10020. https://doi.org/10.18502/ ccb.v1i1.2872

Festi D, Scaioli E, Baldi F, Vestito A, Pasqui F, Di Biase AR, et al. Body weight, lifestyle, dietary habits and gastroesophageal reflux disease. World J Gastroenterol. 2009. 15:1690-1701.

Friedenberg FK, Rai J, Vanar V, Bongiorno C, Nelson DB, Parepally $\mathrm{M}$, et al. Prevalence and risk factors for gastroesophageal reflux disease in an impoverished minority population. Obes Res Clin Pract. 2010. 4:e261-e269.

Gutschow CA, Bollschweiler E, Schröder W, Collet P, Collard JM, Hölscher AH. Effect of "white diet" during bile monitoring with Bilitec 2000 on esophageal pH-metry in patients with gastroesophageal reflux disease. J Gastrointest Surg. 2005. 9:508513.

Hayden JA, Côté P, Bombardier C. Evaluation of the quality of prognosis studies in systematic reviews. Ann Intern Med. 2006. 144:427-437.

Heshmati J, Golab F, Morvaridzadeh M, Potter E, Akbari-Fakhrabadi M, Farsi F, et al. The effects of curcumin supplementation on oxidative stress, Sirtuin- 1 and peroxisome proliferator activated receptor $\gamma$ coactivator $1 \alpha$ gene expression in polycystic ovarian syndrome (PCOS) patients: a randomized placebo-controlled clinical trial. Diabetes Metab Syndr. 2020. 14:77-82.

Heshmati J, Sepidarkish M, Namazi N, Shokri F, Yavari M, Fazelian $\mathrm{S}$, et al. Impact of dietary calcium supplement on circulating lipoprotein concentrations and atherogenic indices in overweight and obese individuals: a systematic review. J Diet Suppl. 2019. 16:357-367.

Ireland CJ, Thompson SK, Laws TA, Esterman A. Risk factors for Barrett's esophagus: a scoping review. Cancer Causes Control. 2016. 27:301-323.

Kahrilas PJ, Shaheen NJ, Vaezi MF; American Gastroenterological Association Institute; Clinical Practice and Quality Management Committee. American Gastroenterological Association Institute technical review on the management of gastroesophageal reflux disease. Gastroenterology. 2008. 135:1392- 
1413, 1413.e1-1413.e5.

Kahrilas PJ. GERD pathogenesis, pathophysiology, and clinical manifestations. Cleve Clin J Med. 2003. 70 Suppl 5:S4-S19.

Kaltenbach T, Crockett S, Gerson LB. Are lifestyle measures effective in patients with gastroesophageal reflux disease? An evidence-based approach. Arch Intern Med. 2006. 166:965971.

Kariri AM, Darraj MA, Wassly A, Arishi HA, Lughbi M, Kariri A, et al. Prevalence and risk factors of gastroesophageal reflux disease in Southwestern Saudi Arabia. Cureus. 2020. 12:e6626. https://doi.org/10.7759/cureus.6626

Keshteli AH, Haghighatdoost F, Azadbakht L, Daghaghzadeh H, Feinle-Bisset C, Afshar H, et al. Dietary glycaemic index and glycaemic load and upper gastrointestinal disorders: results from the SEPAHAN study. J Hum Nutr Diet. 2017. 30:714723.

Khodarahmi M, Azadbakht L, Daghaghzadeh H, Feinle-Bisset C, Keshteli AH, Afshar H, et al. Evaluation of the relationship between major dietary patterns and uninvestigated reflux among Iranian adults. Nutrition. 2016. 32:573-583.

Kim J, Oh SW, Myung SK, Kwon H, Lee C, Yun JM, et al.; Korean Meta-analysis (KORMA) Study Group. Association between coffee intake and gastroesophageal reflux disease: a meta-analysis. Dis Esophagus. 2014. 27:311-317.

Kim YM, Kim JH, Baik SJ, Jung DH, Park JJ, Youn YH, et al. Association between skeletal muscle attenuation and gastroesophageal reflux disease: a health check-up cohort study. Sci Rep. 2019. 9:20102. https://doi.org/10.1038/s41598-019-56702-6

Kubo A, Block G, Quesenberry CP Jr, Buffler P, Corley DA. Dietary guideline adherence for gastroesophageal reflux disease. BMC Gastroenterol. 2014. 14:144. https://doi.org/10.1186/ 1471-230X-14-144

Kubo A, Corley DA, Jensen CD, Kaur R. Dietary factors and the risks of oesophageal adenocarcinoma and Barrett's oesophagus. Nutr Res Rev. 2010. 23:230-246.

Kumar AR, Katz PO. Functional esophageal disorders: a review of diagnosis and management. Expert Rev Gastroenterol Hepatol. 2013. 7:453-461.

Lei WY, Wang JH, Wen SH, Yi CH, Hung JS, Liu TT, et al. Risk of acute myocardial infarction in patients with gastroesophageal reflux disease: a nationwide population-based study. PLoS One. 2017. 12:e0173899. https://doi.org/10.1371/journal. pone.0173899

López-Colombo A, Pacio-Quiterio MS, Jesús-Mejenes LY, Rodríguez-Aguilar JE, López-Guevara M, Montiel-Jarquín AJ, et al. Risk factors associated with gastroesophageal reflux disease relapse in primary care patients successfully treated with a proton pump inhibitor. Rev Gastroenterol Mex. 2017. 82:106-114.

Meining A, Classen M. The role of diet and lifestyle measures in the pathogenesis and treatment of gastroesophageal reflux disease. Am J Gastroenterol. 2000. 95:2692-2697.

Mone I, Kraja B, Bregu A, Duraj V, Sadiku E, Hyska J, et al. Adherence to a predominantly Mediterranean diet decreases the risk of gastroesophageal reflux disease: a cross-sectional study in a
South Eastern European population. Dis Esophagus. 2016. 29: 794-800.

Murphy SJ, Anderson LA, Ferguson HR, Johnston BT, Watson PR, McGuigan J, et al. Dietary antioxidant and mineral intake in humans is associated with reduced risk of esophageal adenocarcinoma but not reflux esophagitis or Barrett's esophagus. J Nutr. 2010. 140:1757-1763.

Nandurkar S, Locke GR 3rd, Fett S, Zinsmeister AR, Cameron AJ, Talley NJ. Relationship between body mass index, diet, exercise and gastro-oesophageal reflux symptoms in a community. Aliment Pharmacol Ther. 2004. 20:497-505.

Newberry C, Lynch K. Can we use diet to effectively treat esophageal disease? A review of the current literature. Curr Gastroenterol Rep. 2017. 19:38. https://doi.org/10.1007/s11894017-0578-5

Rajaie S, Ebrahimpour-Koujan S, Hassanzadeh Keshteli A, Esmaillzadeh A, Saneei P, Daghaghzadeh H, et al. Spicy food consumption and risk of uninvestigated heartburn in Isfahani adults. Dig Dis. 2020. 38:178-187.

Ruhl CE, Everhart JE. Overweight, but not high dietary fat intake, increases risk of gastroesophageal reflux disease hospitalization: the NHANES I epidemiologic followup study. First National Health and Nutrition Examination Survey. Ann Epidemiol. 1999. 9:424-435.

Seremet N, Karaagaoglu N, Kaner G, Tel K. Gastroesophageal reflux symptoms and nutritional preferences. Stud Ethno-Med. 2015. 9:305-318.

Sethi S, Richter JE. Diet and gastroesophageal reflux disease: role in pathogenesis and management. Curr Opin Gastroenterol. 2017. 33:107-111.

Shapiro M, Green C, Bautista JM, Dekel R, Risner-Adler S, Whitacre R, et al. Assessment of dietary nutrients that influence perception of intra-oesophageal acid reflux events in patients with gastro-oesophageal reflux disease. Aliment Pharmacol Ther. 2007. 25:93-101.

Surdea-Blaga T, Negrutiu DE, Palage M, Dumitrascu DL. Food and gastroesophageal reflux disease. Curr Med Chem. 2019. 26: 3497-3511.

Vemulapalli R. Diet and lifestyle modifications in the management of gastroesophageal reflux disease. Nutr Clin Pract. 2008. 23: 293-298.

Winberg H, Lindblad M, Lagergren J, Dahlstrand H. Risk factors and chemoprevention in Barrett's esophagus - an update. Scand J Gastroenterol. 2012. 47:397-406.

Wu KL, Kuo CM, Yao CC, Tai WC, Chuah SK, Lim CS, et al. The effect of dietary carbohydrate on gastroesophageal reflux disease. J Formos Med Assoc. 2018. 117:973-978.

Wu P, Zhao XH, Ai ZS, Sun HH, Chen Y, Jiang YX, et al. Dietary intake and risk for reflux esophagitis: a case-control study. Gastroenterol Res Pract. 2013. 2013:691026. https://doi.org/ $10.1155 / 2013 / 691026$

Yuan L, Tang D, Peng J, Qu N, Yue C, Wang F. Study on lifestyle in patients with gastroesophageal reflux disease. Zhong Nan Da Xue Xue Bao Yi Xue Ban. 2017. 42:558-564. 